\title{
Ampliación de la prevención del VIH basada en pruebas entre niñas adolescentes y mujeres jóvenes [Scaling up evidence- informed HIV prevention for adolescent girls and young women]
}

Melanie Croce-Galis

Karen Hardee

Jill Gay

Follow this and additional works at: https://knowledgecommons.popcouncil.org/departments_sbsr-hiv How does access to this work benefit you? Let us know!

\section{Recommended Citation}

Croce-Galis, Melanie, Karen Hardee, and Jill Gay. 2014. "Ampliación de la prevención del VIH basada en pruebas entre niñas adolescentes y mujeres jóvenes [Scaling up evidence-informed HIV prevention for adolescent girls and young women]," What Works brief. Washington, DC: Population Council and What Works Association, Inc. 


\section{Diciembre 2014}

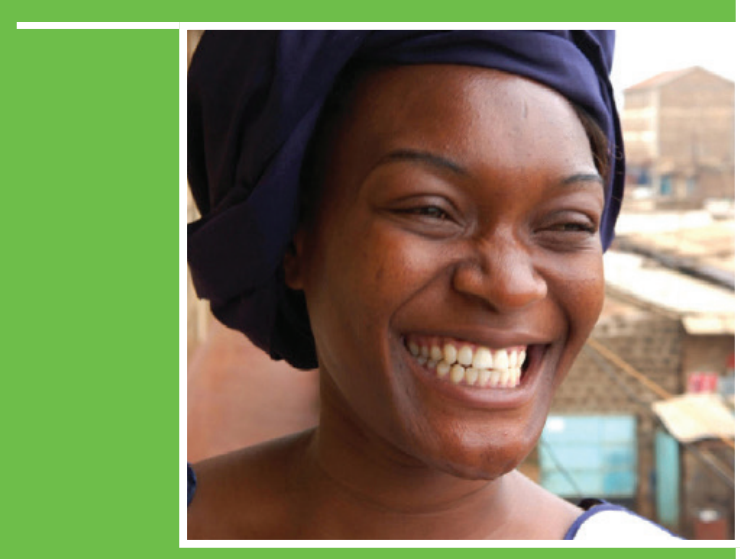

\section{Ampliación de la prevención del VIH basada en pruebas entre niñas adolescentes y} mujeres jóvenes

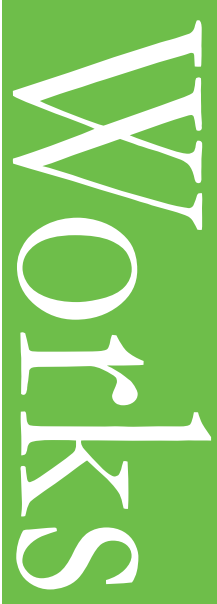

\section{Introducción}

Los adolescentes actuales nunca han conocido un mundo sin SIDA. Las personas nacidas con el VIH y las que inician su actividad sexual en una era de VIH y SIDA están expuestas a complejos riesgos y desafíos que no conocieron las generaciones anteriores. En la actualidad, existen 1.8 billón de jóvenes de 10 a 24 años de edad, el equivalente al $26 \%$ de la población mundial y, en algunas regiones como el África Subsahariana, llegan hasta el 32\%. Muchos de los países donde la prevalencia del VIH es mayor están experimentando un aumento masivo de la población joven, de modo que, aun cuando la prevalencia del VIH se esté reduciendo, el total absoluto de personas que viven con el VIH o en riesgo de contraerlo se incrementará en el próximo lustro. También existen cada vez más pruebas que demuestran que muchas de las conductas de alto riesgo entre las poblaciones clave se inician durante la adolescencia. Por tanto, los adolescentes constituyen una prioridad crucial en la programación de la prevención del VIH. Las mujeres jóvenes son especialmente vulnerables, con unas tasas de infección por VIH que prácticamente duplican a las correspondientes a los hombres jóvenes. A finales de 2012, aproximadamente dos terceras partes de las nuevas infecciones por VIH en adolescentes de 15 a 19 años de

\section{Acerca de este resumen}

El presente resumen ofrece a los programadores intervenciones prioritarias basadas en pruebas de programas satisfactorios dirigidos a mujeres y niñas; si bien, algunas de las intervenciones mencionadas también son beneficiosas para hombres y niños. El resumen se divide en tres partes: áreas prioritarias de programación basadas en pruebas; carencias en materia de investigación y aplicación que han de ser abordarlas; y consideraciones para la ampliación de los programas satisfactorios dirigidos a mujeres y niñas. Puede obtenerse más información acerca de estas y otras intervenciones en www.whatworksforwomen.org. Las intervenciones aquí descritas se basan en una revisión exhaustiva de pruebas globales, pero las respuestas de cada país y comunidad deben adaptarse en función de las oportunidades y los retos locales concretos.

edad afectaron a niñas. Una generación sin SIDA no es posible si no se satisfacen las necesidades concretas que exponen a los adolescentes (especialmente a las niñas) al riesgo de contraer el $\mathrm{VIH}$.
POPULATION COUNCIL Ideas. Evidence. Impact.
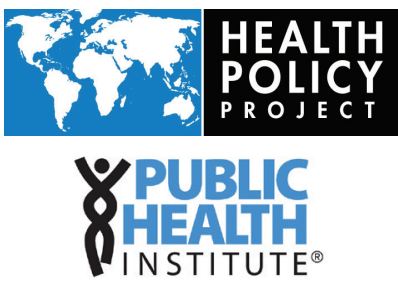

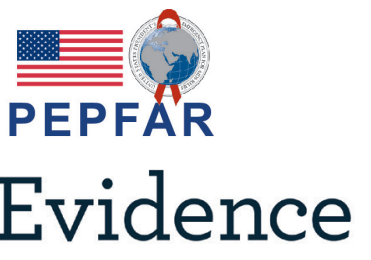




\section{Metodología}

La revisión de la bibliografía de What Works for Women and Girls: pruebas para las intervenciones en materia de VIH/ SIDA (www.whatworksforwomen.org) realizada en enero de 2012 permitió recopilar pruebas de intervenciones eficaces y prometedoras para la prevención del VIH entre mujeres y niñas. Las conclusiones se basaron en reuniones celebradas con expertos destacados en materia de investigación sobre la adolescencia, política y programación, así como en búsquedas realizadas en SCOPUS, Medline y Popline entre 2005 y 2011, con las palabras clave "VIH", "SIDA" "mujer*" y otros términos específicos. La literatura gris se recopiló mediante revisiones de los sitios web principales, entre ellos los de los organismos de las Naciones Unidas, la Organización Mundial de la Salud, la Colaboración Cochrane, el Open Society Institute, el International Council for Research on Women, Population Services International, el Population Council, la Comunidad Internacional de Mujeres Viviendo con VIH/SIDA, el Banco Mundial, FHI 360, AIDStar I y II, y el Instituto Guttmacher, y se consultó con expertos en cada materia para garantizar que las pruebas quedaran plenamente reflejadas (a través de www.whatworksforwomen.org se puede acceder a información más detallada sobre la metodología, incluidos el número total de artículos e informes evaluados y un listado completo de las referencias).
La solidez de las pruebas se baremó con ayuda de una versión modificada de la Escala de Gray (tabla 1), elaborada para las revisiones sistemáticas en el marco de las revisiones sistemáticas de la Colaboración Cochrane (http://www.cochrane.org/) y que se prestaba para las diferentes intervenciones incluidas en esta revisión. La metodología incorpora tres dimensiones para clasificar las intervenciones según dos criterios: "funciona" y "promete": 1) la exhaustividad de las pruebas (cuántas evaluaciones/ estudios respaldan la intervención); 2) la amplitud de las pruebas (cuántos países aportan pruebas para respaldar la intervención); y 3) la solidez de las pruebas (Escala de Gray) donde "funciona" abarca los estudios de tipo I, II o Illa según la Escala de Gray en un mínimo de dos países o cinco estudios de tipo IIIb, IV o V en más de un país; y "promete" abarca los estudios de tipo I, II, o Illa según la Escala de Gray en un único emplazamiento o un mínimo de dos estudios clasificados como IIIb, IV o V en un país o región.

Para la clasificación de las pruebas se utilizó un marco socioecológico y, posteriormente, las pruebas se organizaron en función de una serie de poblaciones de adolescentes y adultos jóvenes. Las categorías son, en cierto modo, fluidas; por ejemplo, las normas de género deben abordarse a nivel comunitario o social; las iniciativas educativas dirigidas a las personas pueden abordarse a nivel social o comunitario, etc. Por tanto, este marco se utiliza a efecto ilustrativo.

\section{Tabla 1. Escala de Gray sobre la solidez de las pruebas}

Tipo

\begin{tabular}{c|l}
\hline I & $\begin{array}{l}\text { Pruebas sólidas de al menos una revisión sistemática de múltiples ensayos controlados aleatorizados, } \\
\text { bien diseñados. }\end{array}$ \\
II & $\begin{array}{l}\text { Pruebas sólidas de al menos un ensayo controlado aleatorizado bien diseñado del tamaño adecuado. } \\
\text { IIla } \\
\text { ejuebas de estudios/ensayos bien diseñados sin aleatorización que incluyen un grupo de control (por } \\
\text { grupo de control). }\end{array}$ \\
IIIb & $\begin{array}{l}\text { Pruebas de estudios/ensayos bien diseñados sin aleatorización que no incluyen un grupo de control } \\
\text { (por ejemplo, estudios de un solo grupo de tipo antes y después, de cohorte, de serie de tiempo/de } \\
\text { serie de tiempo interrumpida). }\end{array}$ \\
IV & $\begin{array}{l}\text { Pruebas de estudios bien diseñados no experimentales de más de un centro o grupo de investigación. } \\
\text { Opiniones de autoridades respetadas, basadas en pruebas clínicas, estudios descriptivos o informes } \\
\text { de comités expertos. }\end{array}$ \\
V
\end{tabular}

Nota: Gray incluye cinco tipos de pruebas. Para What Works, el nivel III se ha subdividido con el fin de diferenciar entre los estudios y las evaluaciones que incluyen grupos de control (IIla) y los que no (IIIb). Los estudios cualitativos pueden pertenecer al nivel IV o V en función, entre otros factores, del número de participantes. 
Principios rectores de los programas dirigidos a los adolescentes

Habida cuenta del carácter formativo tan acusado del período de la adolescencia, es esencial adoptar un enfoque transformador en relación con el sexo (un enfoque concebido para transformar las relaciones entre los sexos con el fin de promover la equidad como un medio para obtener resultados en materia de salud ) en los programas dirigidos a los adolescentes, dado que pueden abordar las normas de género perjudiciales que exponen a las mujeres jóvenes al riesgo de contraer el VIH.

Los programas dirigidos a los adolescentes también deben adherirse a los principios de derechos humanos, tal y como se establece en las convenciones y tratados sobre derechos humanos como la Convención sobre los Derechos del Niño, entre otros.

Cabe señalar que la mayor parte de las pruebas citadas en el presente documento proceden de estudios elaborados con participantes que no necesitaban el consentimiento de los padres para participar en un estudio, es decir, 18 años de edad en numerosos países, aunque pueden existir variaciones (la edad la determina la junta institucional de examen de la investigación de cada país). No obstante, gran parte del trabajo realizado con adultos, como el incremento del asesoramiento y las pruebas, puede ayudar a salvar la vida de los adolescentes. Si bien no todos los estudios citados se realizaron entre adolescentes (se señalan los grupos de edad), algunas conclusiones podrían extrapolarse y, con una rigurosa evaluación, aplicarse a una población adolescente. Del mismo modo, las pruebas sobre la ampli- ación de las intervenciones se basan en experiencias de ampliación de programas para abarcar todas las edades, aunque todavía están surgiendo experiencias específicas con jóvenes y adolescentes. La evaluación constante de la ampliación del alcance de los programas dirigidos a los jóvenes ayudará a subsanar las carencias existentes en las pruebas sobre programas de prevención del VIH dirigidos a los adolescentes.

\section{Intervenciones prioritarias}

A la luz de las pruebas existentes hasta la fecha, las intervenciones que se citan a continuación deberían considerarse áreas prioritarias para la prevención del VIH entre los adolescentes. Los componentes clave de las intervenciones aparecen en cursiva y se señalan las referencias de apoyo. Aunque existen numerosos programas prometedores, son pocas las intervenciones dirigidas a los adolescentes evaluadas exhaustivamente para justificar recomendaciones para su ampliación a escala nacional, con dos notables excepciones: el incremento del nivel educativo de los adolescentes y la impartición de educación integral y de calidad sobre sexualidad.

Nota sobre la edad: las necesidades de los adolescentes muy jóvenes (de 10 a 14 años de edad) difieren enormemente de las de los adolescentes más mayores (de 15 a 19 años de edad) y los adultos jóvenes (de 19 a 24 años de edad), de modo que las intervenciones que se indican a continuación y sus componentes deben aplicarse de un modo adecuado a la edad. No obstante, la conducta sexual de los adolescentes no responde a restricciones de edad: algunos adolescentes pueden iniciar su actividad sexual a una edad temprana, mientras que otros la posponen hasta una edad más avanzada.

\section{Figura 1: Un modelo socioecológico}

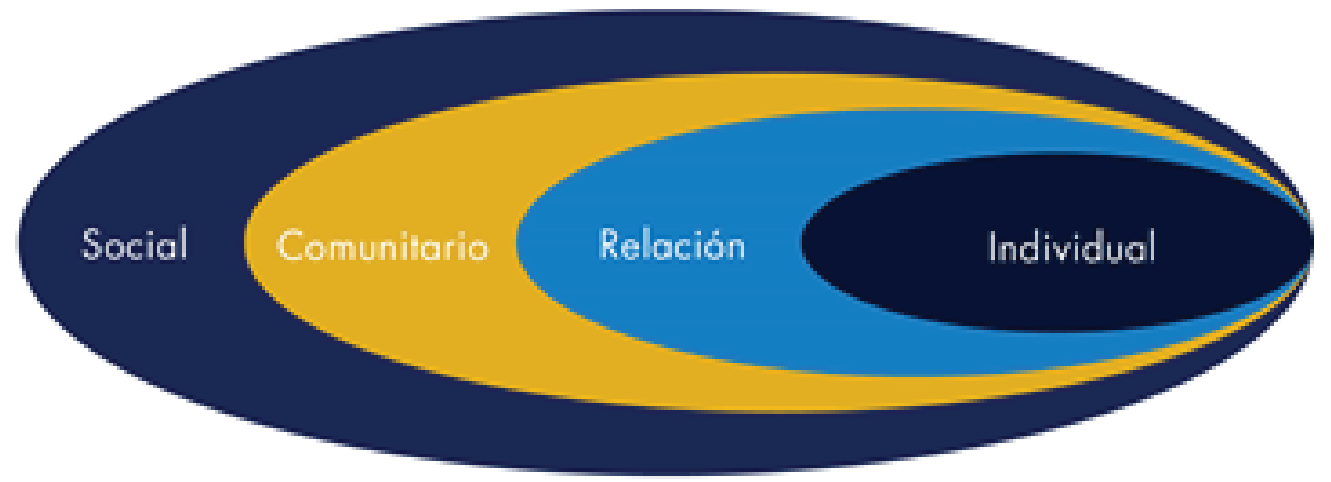

Fuente: Dahlberg LL, Krug EG. Violence - a global public health problem. In: Krug E, Dahlberg LL, Mercy JA, Zwi AB, Lozano R, eds. World Report on Violence and Health. Geneva, Switzerland: World Health Organization; 2002: 1-56. 


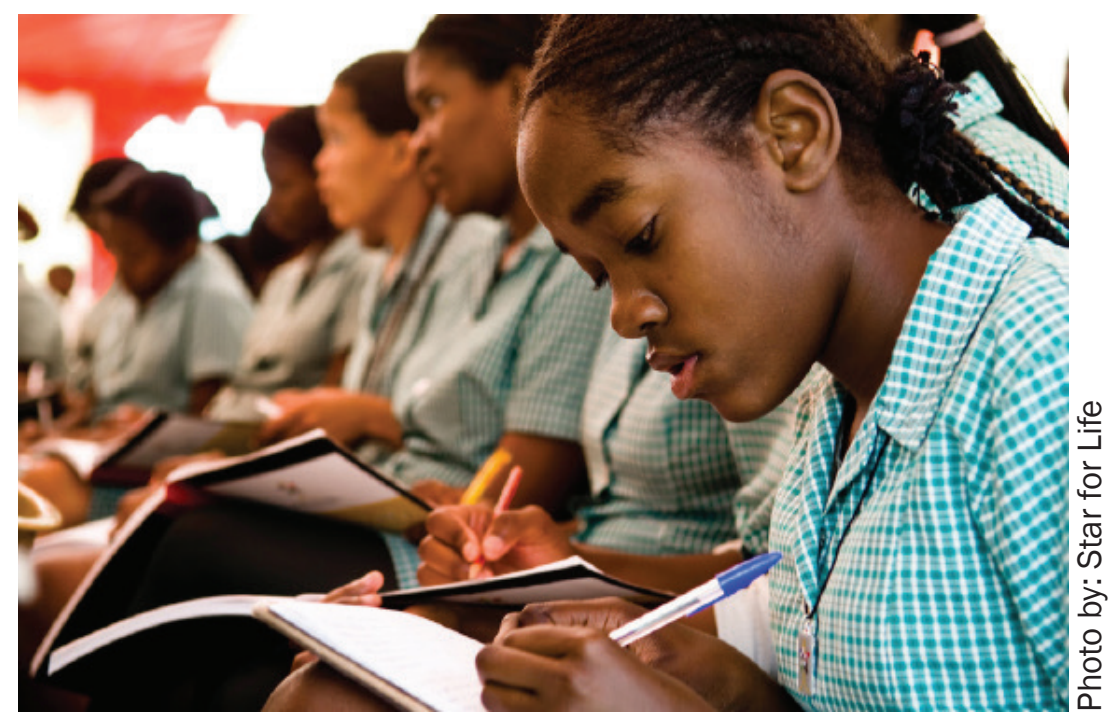

\section{Nivel social}

Funciona: incrementar el nivel educativo de todos los adolescentes

El incremento del nivel educativo de los niños y las niñas puede ayudar a reducir el riesgo del VIH. Existen pruebas sólidas que confirman que el riesgo del VIH es cada vez menor entre los que poseen un nivel educativo más elevado (sobre todo en el caso de las niñas). Algunas intervenciones que lograron incrementar el nivel educativo y que están relacionadas con la reducción de la infección por el VIH son: eliminación del pago de matrículas escolares para potenciar la asistencia de las niñas a (o la permanencia en) la escuela y prestación de apoyo educativo a los huérfanos. Las transferencias monetarias condicionales constituyen una estrategia prometedora para potenciar la permanencia de las niñas en la escuela y reducir la incidencia del VIH.

Las estrategias relacionadas con el VIH deberían promover la escolarización de las niñas. El incremento del nivel educativo exige la colaboración entre los sectores de la salud y la educación, así como los organismos coordinadores del programa sobre el $\mathrm{VIH}$, para la coordinación de la implantación, la supervisión y la evaluación. A escala global, se ha avanzado en el incremento de la finalización de los estudios de educación primaria, pero la garantía de que todos los adolescentes completen la educación secundaria sigue siendo un reto. De por sí, se trata de un objetivo importante y debería tratar de alcanzarse debido a su relación con diferentes resultados positivos en materia de salud, entre ellos el descenso de la incidencia del VIH.
Funciona: impartir educación sexual integral y de calidad a todos los adolescentes

Existen pruebas sustanciales de que la educación sexual y sobre el VIH con determinadas características (por ejempIo, un enfoque claro con respecto a la prevención del VIH, el tratamiento de situaciones susceptibles de provocar relaciones sexuales no deseadas o sin protección, cómo evitarlas y cómo huir de ellas, la atención a la información, los valores, las normas, las actitudes y las habilidades, la utilización de métodos participativos de enseñanza, etc.) antes del inicio de la actividad sexual puede ser eficaz a la hora de reducir el estigma y prevenir la transmisión del VIH, mediante el retraso de la primera experiencia sexual y, en el caso de las personas sexualmente activas, el incremento del uso del preservativo y de las pruebas de detección del $\mathrm{VIH}$, así como la reducción del número de parejas sexuales. Conviene señalar que, por sí solas, las intervenciones en las escuelas no han demostrado ningún impacto en la reducción de la incidencia del $\mathrm{VIH}$, pero han demostrado tener efectos beneficiosos con respecto a los conocimientos y las conductas, lo que sugiere que la educación sexual es necesaria para la prevención eficaz del $\mathrm{VIH}$, pero debe combinarse con otras intervenciones, entre ellas, unos servicios sanitarios accesibles y orientados a los jóvenes.

La calidad de la educación sexual es tan importante como su prestación; debe mantenerse la fidelidad a los componentes eficaces de los programas piloto. Es esencial la formación de los docentes para que impartan una educación sexual participativa y apropiada para cada edad, que mejore los conocimientos y las habilidades de los alumnos. Puede obtenerse información más detallada en Comprehensive Sexuality Education: The Challenges and Opportunities of Scaling Up (UNESCO, 2012). 


\section{Nivel comunitario}

\section{Promete: garantizar el acceso a los servicios sani-} tarios y a las pruebas para todos los adolescentes y adultos jóvenes

Es frecuente que los programas sobre VIH que no están especialmente concebidos para los adolescentes pasen por alto las necesidades de los jóvenes en materia de servicios. La prestación de unos servicios clínicos aceptables y accesibles para los jóvenes, convenientemente situados, asequibles, confidenciales y objetivos constituye una vía prometedora para incrementar la utilización de servicios clínicos de salud reproductiva, incluido el asesoramiento y las pruebas de detección del VIH. Las políticas que exigen el consentimiento de los padres para el asesoramiento y las pruebas de detección del VIH pueden impedir que los adolescentes accedan a los servicios. Existen pruebas que demuestran que el asesoramiento y las pruebas de detección del VIH puede ayudar a las mujeres a conocer su estado serológico y reforzar su conducta de protección, especialmente entre las que obtienen resultados positivos (funciona en adultos), si bien son pocos los estudios de pruebas orientados a los adolescentes. Se ha demostrado que el asesoramiento y las pruebas de detección del VIH junto con otros servicios sanitarios aumenta el número de adultos que acceden a asesoramiento y pruebas para la detección del VIH (funciona en adultos). En el caso de los adolescentes que viven con el $\mathrm{VIH}$, un tratamiento antirretrovírico puede reducir (pero no eliminar) el riesgo de transmisión del VIH y constituye una estrategia adicional de prevención del VIH. El establecimiento de unos protocolos integrales de atención a las víctimas de violación, que incluyan profilaxis después de la exposición y anticonceptivos de emergencia, puede mejorar los servicios para todos.

Promete: empleo de las campañas de mercadotecnia social y los medios de comunicación para llegar a los adolescentes

Las campañas de mercadotecnia social y de los medios de comunicación han sido herramientas útiles para la consecución de una serie de objetivos y, si se utilizan de manera centrada y coordinada con otras intervenciones, pueden llegar de forma eficaz a un gran número de jóvenes para incrementar el número de personas y parejas que acceden al asesoramiento y las pruebas de detección del VIH. , Estas campañas han sido moderadamente eficaces a la hora de convencer a los adolescentes, tanto varones como mujeres, para que cambien sus conductas de riesgo. En adultos, las pruebas han demostrado que las intervenciones basa- das en la comunidad (incluidos los medios) que transmiten información precisa sobre la transmisión del VIH pueden reducir considerablemente el estigma del VIH y la discriminación (funciona en adultos); las campañas de los medios de comunicación concernientes a la igualdad de género en el marco de intervenciones integrales e integradas pueden incrementar las conductas de protección relacionadas con el VIH (promete en adultos); y la promoción de la salud pública puede incrementar la concienciación con respecto a la violencia contra las mujeres (promete en adultos).

\section{Nivel de relaciones}

Promete: atención a la equidad entre hombres y mujeres para todos los adolescentes y adultos jóvenes

Las normas de género perjudiciales son un factor de riesgo de violencia y pueden agravar el riesgo de contraer el VIH. Los expertos en cuestiones de género y desarrollo cada vez están más de acuerdo en que las intervenciones para tratar las normas de género y reducir el VIH tienen que funcionar con "hombres y mujeres, niños y niñas, de un modo intencional y complementario que cuestione las normas de género, catalice los logros alcanzados en materia de igualdad de género y mejore la salud". El tratamiento de la equidad entre hombres y mujeres durante el período formativo de la adolescencia puede transformar las actitudes de por vida.

La formación de los docentes con respecto a la violencia de género constituye una estrategia prometedora para cambiar las normas relativas a su aceptación. En el caso de los adolescentes de mayor edad y los adultos jóvenes (de 16 a 24 años) los enfoques de aprendizaje participativos basados en la comunidad en los que intervienen hombres y mujeres pueden crear relaciones que reflejen mejor la equidad de género, con la consiguiente reducción de la violencia (funciona en adultos). Existen pruebas que demuestran que el tratamiento de la igualdad de género, en particular entre los hombres (sin especificación de edad), con formación, conversaciones entre iguales y con la pareja, y educación basada en la comunidad que cuestionen las normas de género perjudiciales, puede mejorar la prevención del VIH, las pruebas para su detección, el tratamiento y la atención (funciona en adultos). En adultos, el diálogo y el asesoramiento de la pareja, incluidas técnicas para evitar la violencia de género, también pueden incrementar el número de parejas que reciben y divulgan los resultados de sus pruebas (funciona en adultos). Esto podría probarse también a modo de estrategia dirigida a los adolescentes casados y los que tengan pareja. 


\section{Nivel individual}

\section{Promete/funciona: mejorar los conocimientos y fo-} mentar una conducta de protección entre los adolescentes más jóvenes

Las iniciativas nacionales concebidas para reducir o demorar la actividad sexual, incrementar el uso del preservativo y reducir el número de parejas sexuales pueden resultar eficaces para la prevención de infección por el $\mathrm{VIH}$. Entre las intervenciones satisfactorias figuran los programas integrales dirigidos a los jóvenes, como los que se sirven de varios componentes (por ejemplo, la política/la promoción de la coordinación y la integración de los servicios sanitarios, la formación sobre técnicas de subsistencia, seminarios, deportes, etc.), que pueden mejorar los conocimientos de los adolescentes en relación con el VIH y fomentar una conducta de protección (funciona). El asesoramiento puede reducir las conductas de riesgo y la infección por el VIH entre los adultos, y el hecho de incluir análisis acerca del consumo de alcohol en el asesoramiento y las pruebas de detección del VIH puede incrementar las conductas de protección tales como el uso del preservativo, la reducción de parejas, y la reducción del consumo de alcohol (funciona en adultos), si bien estos estudios no se han testado expresamente entre adolescentes. Intervenciones prometedoras como el fomento de la comunicación entre adultos y jóvenes con respecto a la información relativa a la salud reproductiva pueden incrementar las conductas de protección entre los adolescentes, y la educación, por parte de personas que viven con el VIH, relativa a la prevención del VIH (personas a las que no les importe dar a conocer su estado serológico) destinada a los jóvenes puede reforzar los mensajes acerca de la conducta de protección.

\section{Promete: atención a los preservativos para los adoles- centes sexualmente activos}

El uso del preservativo constituye un método de prevención crucial que reduce la posibilidad de contraer el VIH en más de un $95 \%$, siendo la eficacia de los preservativos masculinos y femeninos equiparable cuando se utilizan de forma constante y correcta. Desde el momento en que se inicia la actividad sexual, el fomento del preservativo, en sesiones individuales o de grupo, junto con la formación, el suministro de preservativos y la educación motivadora pueden incrementar el uso del preservativo (funciona en adultos). El incremento de la accesibilidad y disponibilidad de preservativos puede incrementar el uso del preservativo

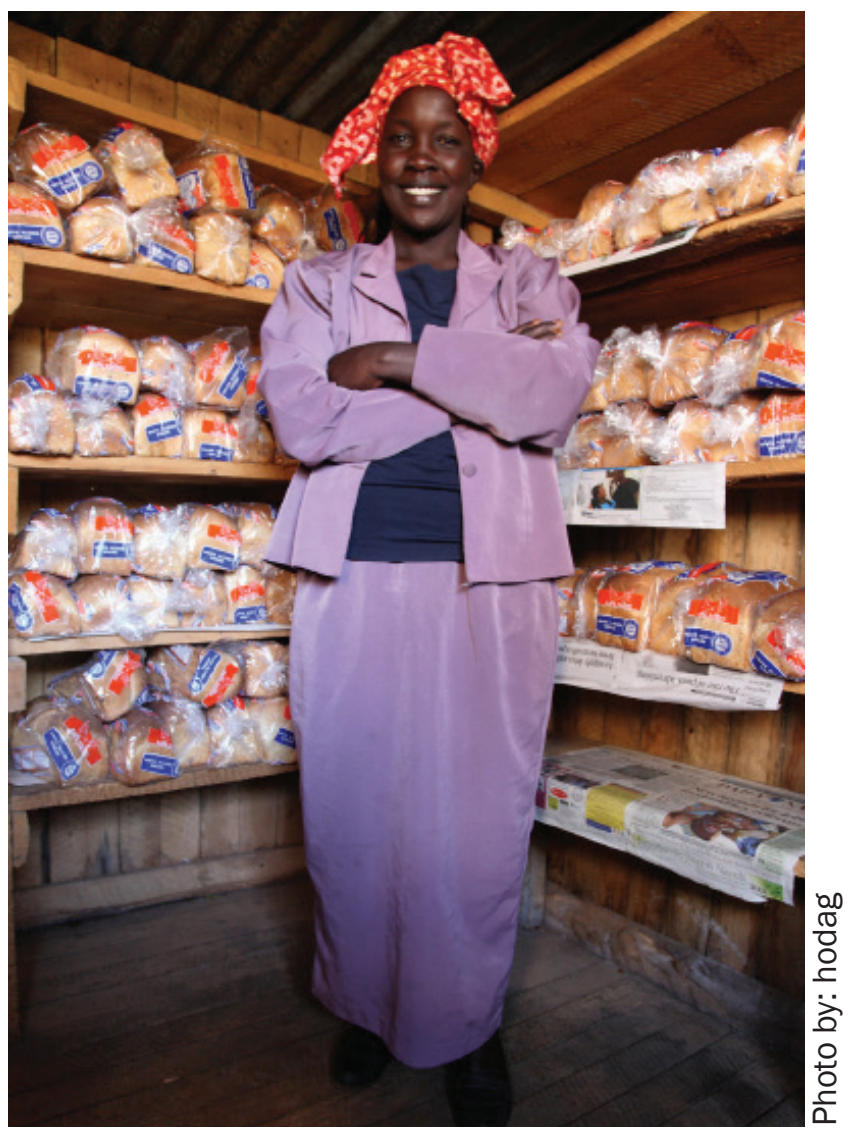

(promete en adultos). Asimismo, el aumento de la distri bución de preservativos femeninos puede incrementar el uso de este preservativo, con el consiguiente incremento del número de actos sexuales seguros y de la prevención de la contracción y la transmisión del VIH (funciona en adultos). El fomento del doble uso del preservativo como anticonceptivo y para la prevención del VIH puede hacer que su empleo resulte más aceptable (funciona en adultos), , fundamentalmente para los jóvenes (promete en adolescentes). Además, la distribución de preservativos orientada a los jóvenes puede ayudar a que los adolescentes se sientan más cómodos a la hora de acceder a ellos (promete en adolescentes) y el fomento del placer asociado al uso del preservativo masculino y femenino puede incrementar la práctica de un sexo más seguro (promete en adultos). En lo que respecta a los adolescentes de mayor edad, las intervenciones eficaces incluyen también el incremento de la comunicación entre la pareja con respecto al riesgo del $\mathrm{VIH}$, lo que puede incrementar las conductas de prevención, incluido el uso del preservativo (funciona en adultos). En términos generales, el fomento de la aceptación del uso del preservativo por parte tanto de las mujeres como de los hombres por norma para las relaciones sexuales constituye una estrategia prometedora para reducir la prevalencia nacional del $\mathrm{VIH}$. 


\section{Promete: propiciar el empleo para adolescentes de} mayor edad y adultos jóvenes

Aunque se necesitan mejores indicadores y programas diseñados con cautela para medir el impacto relacionado con el VIH y el SIDA del fortalecimiento económico sobre mujeres y niñas, los estudios realizados demuestran invariablemente que el incremento del acceso de la mujer a la información, el conocimiento, las tecnologías, los servicios, el apoyo social y los ingresos incrementó su capacidad para protegerse a sí mismas del VIH. En el caso de los hombres jóvenes sucede lo mismo: el mayor acceso al empleo propicia un mayor desarrollo social y humano y la erradicación de la pobreza. Mayores oportunidades de empleo, microfinanciación o actividades que generan ingresos a pequeña escala también pueden reducir la conducta que agrava el riesgo del $\mathrm{VIH}$, fundamentalmente entre los adultos, y puede resultar prometedor entre los adolescentes y los adultos jóvenes si se evalúa detenidamente. , La disponibilidad de asesoramiento y pruebas de detección del VIH en los lugares de trabajo podría incrementar el porcentaje de asesoramiento y pruebas de detección del VIH.

\section{Funciona: apoyo a los huérfanos y niños vulnerables}

Los huérfanos y los niños vulnerables representan un sector de la población clave con respecto al riesgo de contraer el VIH. Las niñas que han quedado huérfanas a causa del VIH y el SIDA son objeto de un compendio de vulnerabilidades: por su temprana edad, carecen de derechos legales (por ejemplo, sucesorios y de propiedad), de la madurez y de las aptitudes para cuidar de sí mismas y ser autosuficientes. Por ser niñas, no suelen gozar de igualdad de oportunidades para acceder a recursos domésticos para la escolarización, la nutrición o la atención sanitaria. Ante una situación de catástrofe para la familia, suelen ser las niñas las que deben abandonar la escuela y asumir una carga mayor en el hogar.

Entre las intervenciones satisfactorias dirigidas a los huérfanos y los niños vulnerables cabe destacar el apoyo psicológico y el tutelaje, que pueden mejorar su bienestar psicológico. Los programas que ofrecen apoyo social, sanitario y económico integrado y centrado en la familia, también pueden ofrecer mejores resultados en materia de educación y salud para los huérfanos. Puesto que estos últimos están expuestos a un riesgo de VIH particular, los programas que ofrecen transferencias de efectivo para el conjunto de la comunidad, oportunidades de microempresas, pensiones $u$ otros tipos de asistencia financiera y para la obtención de medios de subsistencia pueden ayudar a los huérfanos de un modo eficaz. Entre las estrategias prometedoras dirigidas a los huérfanos y los niños vulnerables figura la creación de proyectos de desarrollo comunitario, en lugar de un programa de VIH y SIDA poco definido, para reducir el estigma contra esos huérfanos a causa del SIDA.

\section{Promete/funciona: ayudar a las mujeres jóvenes que se dedican al trabajo sexual a protegerse}

Aunque las mujeres jóvenes pueden acceder al trabajo sexual a edad temprana, "las Naciones Unidas restringen su definición de 'trabajador sexual' a los adultos de más de 18 años de edad y afirman que la participación de niños (menores de 18 años) en relaciones sexuales transaccionales/trabajo sexual u otras formas de explotación y abuso contravienen las convenciones de las Naciones Unidas y el derecho internacional de los derechos humanos. Los niños menores de 18 años que venden servicios sexuales son víctimas y no pueden considerarse trabajadores sexuales". Las intervenciones de prevención del VIH dirigidas a niños explotados y víctimas de la trata quedan fuera del alcance de la presente revisión.

En el caso de las trabajadoras sexuales mayores de 18 años, algunas intervenciones programáticas han demostrado buenos resultados, entre ellas los programas de prevención integrales que incluyen componentes como la educación por pares, servicios médicos y suministros, y grupos de apoyo. Todo ello puede resultar eficaz para ayudar a los trabajadores sexuales a adoptar prácticas sexuales con menos riesgo (funciona en adultos). La educación por pares entre los trabajadores sexuales (funciona en adultos) y las políticas que tienen en cuenta a los trabajadores sexuales, propietarios de burdeles y clientes en el desarrollo y la aplicación del uso del preservativo pueden aumentar los datos de dicho uso (promete en adultos). ,

Otras intervenciones satisfactorias son: intervenciones basadas en clínicas con agentes de planificación familiar, que pueden resultar eficaces a la hora de incrementar el uso del preservativo y las pruebas de detección del VIH entre los trabajadores sexuales (funciona en adultos); la prestación de servicios clínicos rutinarios, de gran calidad, voluntarios y confidenciales sobre infecciones de transmisión sexual que incluyen el fomento del preservativo pueden reducir el riesgo del VIH entre los trabajadores sexuales, (promete en adultos) y la creación de un sentimiento de comunidad, capacitación y liderazgo entre los trabajadores sexuales puede ayudar a respaldar una prevención eficaz 
del VIH (funciona en adultos). La orientación a los clientes masculinos es una intervención prometedora que puede incrementar el uso del preservativo y reducir el riesgo del $\mathrm{VIH}$ entre los trabajadores sexuales.

\section{Promete: fomento de una conducta de protección en-} tre los jóvenes que se inyectan drogas

En las zonas donde el consumo de drogas contribuye a la epidemia del VIH, sobre todo entre los adolescentes de mayor edad y los adultos jóvenes, el tratamiento con agonistas opioides, fundamentalmente los programas de mantenimiento con metadona y buprenorfina, provocan una reducción del consumo de drogas, la infección por el $\mathrm{VIH}$ y las conductas de riesgo entre los usuarios de drogas inyectables, y es segura y eficaz para las mujeres embarazadas (funciona en adultos). Los programas integrales de reducción del daño, incluidos los programas de intercambio de agujas, la distribución de preservativos, el tratamiento con agonistas y la difusión, las intervenciones lideradas por pares, y el asesoramiento objetivo para la reducción de riesgos pueden reducir las conductas de riesgo de infección por el VIH y su prevalencia entre los usuarios de drogas inyectables (funciona en adultos).

El fomento del uso del preservativo y otras conductas de protección es importante para los jóvenes que consumen drogas. Entre las intervenciones que refuerzan la conducta de protección figuran la educación por pares entre mujeres que consumen drogas y las parejas femeninas de hombres que consumen drogas (funciona en adultos). Las sesiones en grupos segregados en función del sexo y que tienen en cuenta las cuestiones de género y están dirigidas a parejas que consumen drogas también pueden incrementar el uso del preservativo y las prácticas de inyección más seguras (funciona en adultos). La prestación de asesoramiento y pruebas de detección del VIH gratuitos a las mujeres que consumen drogas es una intervención prometedora que puede ayudar a reducir las conductas de riesgo de infección por el VIH.

\section{Carencias en materia de apli- cación e investigación en las pruebas correspondientes a los adolescentes}

Las intervenciones que generan un entorno positivo y brindan unos servicios adecuados y accesibles aportan a los adolescentes los conocimientos y los recursos necesarios para protegerse a sí mismos y a sus parejas. Pero sigue existiendo una serie de carencias en materia de aplicación e investigación y, en términos generales, es necesario ampliar los programas eficaces para que lleguen a más adolescentes, en especial a los más desatendidos, como los adolescentes muy jóvenes, los jóvenes que no asisten a la escuela, los jóvenes que viven con el $\mathrm{VIH}$, aquellos sin hogar y los que viven en zonas rurales. Las carencias que se indican a continuación se ha detectado mediante una revisión exhaustiva de la bibliografía.

\section{Nivel social...}

Para reducir el riesgo de transmisión del VIH entre los jóvenes se precisan unas políticas y una legislación claras que respalden el acceso a la información y los servicios. Las leyes y las prácticas que obstaculizan el acceso de los adolescentes a los servicios, como el consentimiento de los padres, la edad, los requisitos relativos al estado civil, deben examinarse y revisarse. Deben promulgarse y aplicarse leyes que prohíban el matrimonio a una edad temprana. Las iniciativas satisfactorias concebidas para incrementar el nivel educativo de las niñas, en particular en la educación secundaria, deben fomentarse.

\section{Nivel comunitario...}

Se necesitan intervenciones concebidas para reducir las relaciones sexuales y los matrimonios intergeneracionales, así como las que se proponen eliminar la coerción sexual y la violación de mujeres, niñas, niños y hombres. Se necesitan iniciativas para concienciar a las comunidades de que la violencia contra adultos y niños es inaceptable, reforzar los sistemas legales de protección de los adultos y los niños, y conceptualizar e instaurar unos servicios apropiados para la protección de los adultos y los niños en los países en desarrollo, vinculando los programas sobre el VIH y el SIDA con programas que aborden la violencia por razón de género, incluidos el abuso sexual de menores y la profilaxis después de la exposición.

En las zonas con elevadas tasas de consumo de drogas intravenosas, se necesitan intervenciones que fomenten e incrementen el acceso a la metadona y la buprenorfina (tratamiento con agonistas eficaz para la drogodependencia) así como programas sobre distribución/intercambio de agujas. También se necesitan iniciativas para eliminar la detención obligatoria de los drogodependientes y ofrecer a cambio a los usuarios de drogas inyectables unos servicios de pruebas y prevención del VIH y un tratamiento eficaz de la drogodependencia de la mano de profesionales médicos. 
Las adolescentes que se inyectan drogas requieren programas de reducción de los riesgos que cubran sus necesidades, y deberían existir servicios de información sobre prevención del VIH y confidenciales para los usuarios de drogas inyectables que estén recibiendo tratamiento para el consumo de sustancias. Se necesitan intervenciones que ayuden a las mujeres y a sus parejas a comprender mejor el riesgo de contraer el VIH mediante las prácticas sexuales y el consumo de drogas inyectables. Las mujeres que se inyectan drogas deben poder acceder a servicios para la violencia contra las mujeres, el uso del método de doble protección, los anticonceptivos seguros y la salud reproductiva. Muchas de estas carencias se han observado entre los adultos, pero también constituyen carencias entre los adolescentes.

Los programas de educación sexual y promoción del preservativo deben considerar las distintas motivaciones que llevan a los hombres y las mujeres jóvenes a mantener relaciones sexuales sin protección y los programas sobre el VIH deben ser más eficaces a la hora de promover una participación significativa por parte de las parejas masculinas y femeninas y una atención cada vez mayor a la igualdad de género. También es preciso incrementar la formación dirigida a los docentes y unas políticas educativas claras con respecto a la educación sexual para ofrecer una educación eficaz sobre el SIDA.

Deberían adoptarse medidas para mejorar los conocimientos de los adolescentes acerca de cuándo y dónde acceder a los servicios sanitarios, incluido el acceso a servicios de control de la natalidad y a preservativos. Existe una necesidad imperiosa de intervenciones personalizadas para ofrecer mayor disponibilidad y acceso a los preservativos femeninos, junto con educación y formación sobre su uso como opción adicional al condón. Debería formarse a los proveedores para que promuevan el uso de preservativos femeninos y hablen sobre sexualidad y placer con los adolescentes que utilicen los servicios de salud reproductiva.

Deben proseguir los programas que promuevan una conducta de protección, como el uso del preservativo, así como la circuncisión masculina. Los programas relativos a la circuncisión masculina deberían facilitar, tanto a mujeres y niñas, como a hombres y niños, datos empíricos detallados sobre las ventajas y los riesgos de la circuncisión masculina médica voluntaria e incluir formación en cuestiones de género para los hombres jóvenes.

Se necesitan nuevas iniciativas para poner el asesoramiento y las pruebas de detección del VIH a disposición y

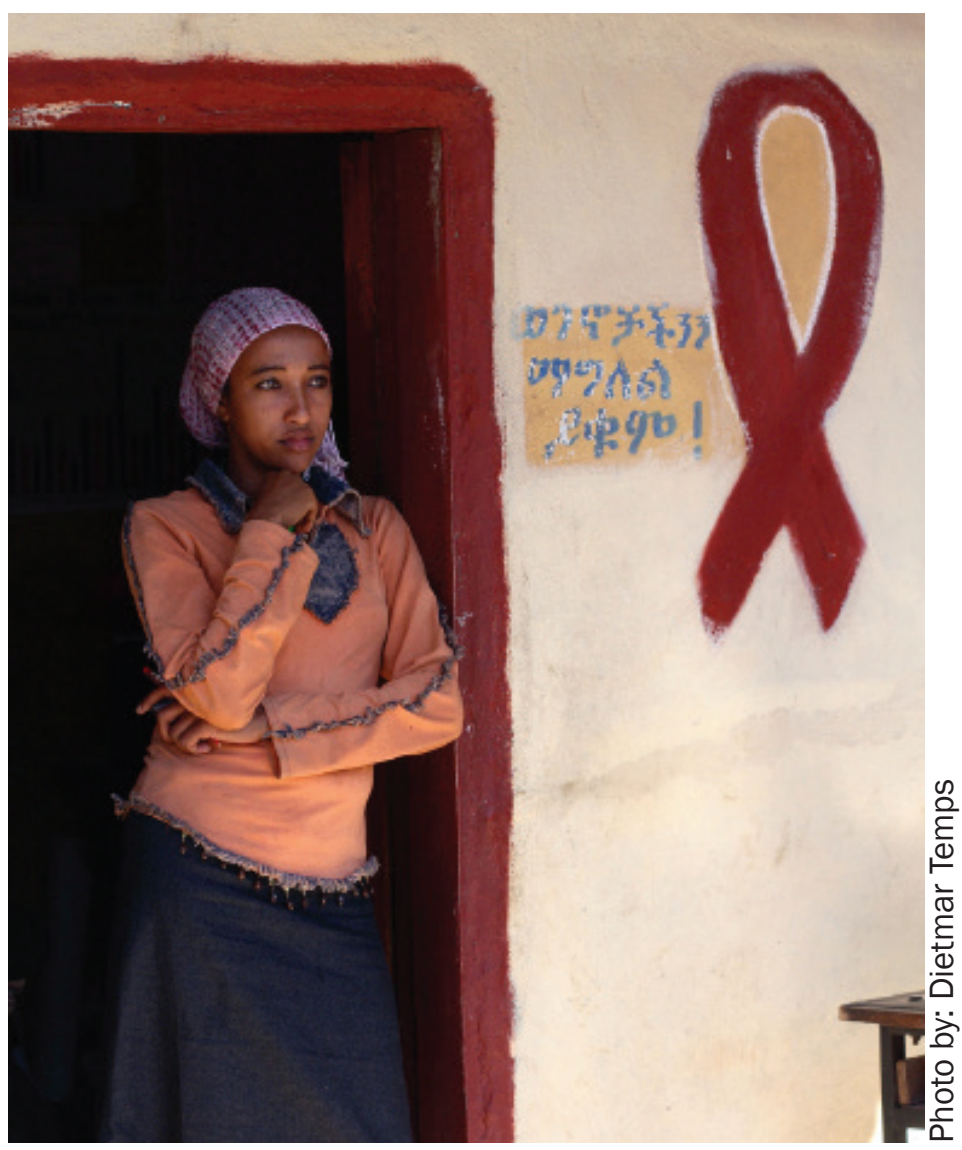

accesibles para los adolescentes y a fin de garantizar la confidencialidad de los resultados las pruebas de detección del VIH. Asimismo, deben darse los pasos necesarios para garantizar unas estrategias y unos temas de asesoramiento y pruebas de detección del VIH óptimos, con información detallada acerca del acceso al tratamiento y la reducción del riesgo. La aplicación de los protocolos ordinarios es necesaria para reducir el riesgo de coacción por parte del proveedor en las pruebas de detección del VIH, en particular en las pruebas y el asesoramiento a cargo del proveedor.

\section{Nivel de relaciones...}

El cambio de las normas de género que pueden derivar en el incremento del riesgo de contraer el $\mathrm{VIH}$, como las que imponen relaciones sexuales con múltiples personas, el uso de la violencia o un consumo abusivo de la bebida para los hombres e ignorancia sexual, sumisión y dependencia para las mujeres y las niñas supone un reto., Se necesitan intervenciones nuevas bien evaluadas y es necesario ampliar las intervenciones existentes. Se necesitan intervenciones para reducir la homofobia, que puede llevar a hombres que tienen relaciones sexuales con hombres a mantener relaciones sexuales con mujeres (un motivo de 
especial preocupación para los jóvenes, pues la identidad y la exploración sexual se desarrollan durante la infancia y la adolescencia). La expansión y el fomento de las intervenciones que promueven oportunidades económicas para las mujeres y las niñas son necesarias para incrementar su capacidad para rechazar las relaciones sexuales sin protección motivadas por necesidades económicas. Las intervenciones que reducen el tiempo de desplazamiento al lugar de trabajo o garantizan un acceso fácil y seguro al transporte público pueden reducir el riesgo de violencia sexual entre las adolescentes.

Existe una necesidad imperiosa de intervenciones evaluadas para reducir las relaciones múltiples y simultáneas (en particular cuando el riesgo percibido de VIH es reducido, las mujeres están sometidas a normas de género que abogan por la fidelidad y los hombres están sometidos a normas de género que abogan por las relaciones sexuales con múltiples personas).

\section{Nivel individual...}

Se requieren mayores esfuerzos para ayudar a los jóvenes a personalizar los riesgos del VIH y para que los adolescentes acepten menos la violencia de género y el estigma contra las personas que viven con el VIH.

Se necesitan intervenciones para educar a las niñas de 8 a 14 años en lo relativo a la menstruación, la pubertad y conocimientos básicos sobre el VIH.

Tabla 2. Diez factores facilitadores clave para la ampliación 118

1. Simplicidad técnica de la intervención

2. Percepción de demanda de la intervención

3. Recursos disponibles para la ampliación del alca nce

de las experiencias piloto prometedoras

4. Adaptabilidad a distintos entornos

5. Sistemas listos para la ampliación de su alcance

6. Compromiso político

7. Reforzado mediante políticas

8. Incluye a todas las partes interesadas

9. Mecanismos de rendición de cuentas instaurados/ desarrollados

10. Consideraciones de género y derechos humanos abordadas
Se necesitan nuevas intervenciones para ayudar a las niñas huérfanas y vulnerables a reducir las conductas sexuales de riesgo y protegerlas de la violencia sexual. Se necesitan programas de apoyo, incluido asesoramiento, para que los niños huérfanos a causa del SIDA y sus cuidadores combatan la depresión, el aislamiento social y el estigma, y los programas deberían fomentar la participación masculina en el tratamiento de los niños y el cuidado de los huérfanos.

Para los adultos jóvenes que sean trabajadores sexuales (véase la definición de las Naciones Unidas más arriba), todavía se requiere información básica sobre los servicios relacionados con el $\mathrm{VIH}$, como dónde acceder a los preservativos y a pruebas confidenciales de detección del VIH.

La atención a estas carencias en materia de investigación y aplicación es vital para el desarrollo de una completa base de pruebas de estrategias de prevención del VIH eficaces para los jóvenes.

\section{El camino a seguir: consid- eraciones para la ampliación de los programas satisfactorios di- rigidos a los adolescentes}

El fomento de la prevención entre los adolescentes es importante para llegar a más jóvenes. Existen numerosas iniciativas probadas a pequeña escala para llegar a los jóvenes, pero, si no se amplía su escala, no repercutirán en la incidencia y la prevalencia del VIH. En una revisión de la investigación realizada en materia de prevención del VIH en los últimos 25 años, Rotheram-Borus, et al. (2009) reflejaba que "la prevención eficaz del VIH pasa por una combinación de estrategias de intervención conductuales, biomédicas y estructurales", pero la ampliación de las intervenciones probadas ha sido insuficiente. Señalan que el reto actual para los investigadores dedicados a la prevención consiste en "reconceptualizar el modo en que se diseñarán, ofrecerán, probarán y difundirán los programas de prevención rentables, útiles, realistas y sostenibles".

Globalmente existen enormes variaciones entre los adolescentes y, para tener éxito, los programas tendrán que ser, necesariamente, específicos para cada contexto. Sin embargo, la bibliografía demuestra que la prevención del VIH entre los adolescentes en general pasa por una respuesta 
integral y multisectorial con la repetición de mensajes coherentes en numerosas ocasiones y desde múltiples fuentes. Para ampliar las intervenciones eficaces se necesitan una serie de factores clave (tabla 2).

En los niveles político, programático, de prestación de servicios y comunitario también se ha identificado una serie de limitaciones que pueden impedir que se avance en la ampliación. En los programas de ampliación dirigidos a los adolescentes han tenerse en cuenta tanto los factores facilitadores como las limitaciones. Por ejemplo, entre los obstáculos con los que se encuentran los adolescentes a nivel doméstico/comunitario se incluye el permiso de los padres para las pruebas de detección del VIH; entre los obstáculos en el nivel de prestación de servicios sanitarios se incluye la falta de privacidad e infraestructuras centradas en los adolescentes (la burocracia gubernamental suele pasar por alto a los adolescentes y las necesidades de información de los jóvenes no son prioritarias).

Como han señalado Mavedzenge, et al. (2013) en su revisión sistemática de revisiones sistemáticas, existen pruebas sólidas de intervenciones relacionadas con el VIH concebidas fundamentalmente para los adultos, y tan solo existe un modesto número de pruebas de cómo podrían acceder los adolescentes a dichas intervenciones. No obstante, se pueden ampliar algunos programas basados en pruebas para llegar a más adolescentes, sobre todo niñas. Existen pruebas sólidas que confirman que el incremento del nivel educativo, especialmente en la educación secundaria, ayuda a reducir el riesgo de infección por el VIH entre las niñas y está asociado a otros beneficios económicos y positivos para la salud. Debería ampliarse el alcance de las intervenciones probadas que facilitan la permanencia de las niñas en las escuelas, como las transferencias de efectivo y la eliminación del pago de matrículas escolares, en el marco de las estrategias nacionales del VIH para dirigirlas a las niñas que se encuentran en mayor riesgo de obtener unos resultados deficientes relativos al VIH. La educación sexual integral y de alta calidad es una intervención con amplias pruebas que demuestran su idoneidad para que se amplíe su alcance en todos los países, con esfuerzos adicionales necesarios para jóvenes no escolarizados.

Otros enfoques prometedores para la ampliación incluyen la garantía de acceso a servicios e información sanitaria, la mejora de los conocimientos sobre el VIH/SIDA en función de la edad entre los adolescentes más jóvenes, las iniciativas centradas en la información sobre el preservativo y el suministro de los mismos a los adolescentes sexualmente activos, y las oportunidades de empleo entre los adolescentes de mayor edad. La atención a la equidad de género para todos los adolescentes durante sus años formativos también puede tener un impacto crucial en la igualdad de género en un futuro cercano. Se han aplicado intervenciones prometedoras para grupos especialmente vulnerables como los niños huérfanos y vulnerables, los usuarios de drogas inyectables o los trabajadores sexuales.

Siguen existiendo importantes carencias en lo referente a la investigación, programación y evaluación de la experiencia de ampliación de programas para niñas. Un programa satisfactorio para mujeres adultas podría no ser adecuado para niñas adolescentes. Es necesario realizar rigurosas evaluaciones, en especial en los enfoques satisfactorios aplicados ente los adultos cuya ampliación podría considerarse con el fin de llegar a los adolescentes. También se necesita información adicional sobre los costos y la rentabilidad. Las pruebas aquí presentadas ofrecen orientaciones útiles sobre intervenciones potenciales que, tras una exhaustiva evaluación, podrían ampliarse para llegar al número, cada vez mayor, de adolescentes, especialmente de niñas, que viven con el VIH o en riesgo de contraerlo.

Pude obtenerse más información sobre los adolescentes, una descripción detallada de la metodología y referencias completas sobre las citas en www.whatworksforwomen.org.

Cita sugerida: Croce-Galis, M; Hardee, K; Gay, J (2014). Scaling up Evidence-Informed HIV Prevention for Adolescent Girls and Young Women. Washington DC: Population Council, Evidence Project. www.whatworksforwomen.org

Las fotografías de esta publicación son para fines ilustrativos y no representan ninguna salud o condición sexual de los sujetos de fotos.

\section{Referencias}

1. Fransen-dos-Santos, R. 2009. "Young People, Sexual and Reproductive Health and HIV." Bulletin of the World Health Organization 87 (11): 877-879.

2. United Nations, Department of Economic and Social Affairs, Population Division (2013). World Population Prospects: The 2012 Revision, CD-ROM Edition.

3. IOM. 2011. The Science of Adolescence Risk-Taking: Workshop Report. Washington, DC: National Academy Press.

4. UNAIDS. 2011. UNAIDS World AIDS Day Report 2011.

5. UNICEF. 2013. Towards an AIDS-Free Generation: Children and AIDS - Sixth Stocktaking Report. NY.

6. Gray, 1997, Evidence Based Health Care: How to Make Health Policy and Management Decisions. London, UK: Churchill Living- 
stone.

7. Puede obtenerse más información acerca de estos tipos de estudios y sus puntos fuertes y sus puntos débiles en Gray, 2009, Evidence-Based Health Care and Public Health: How to Make Decisions About Health Services and Public Health. 3rd Edition. Edinburgh, Scotland: Churchill Livingston Elsevier.

8. Interagency Gender Working Group (IGWG). (2013). Gender Terms and Definitions; Gender Continuum: http://www.igwg.org/ igwg_media/integrgendrRH-HIV/gendertermsdefinitions.pdf 9. (Office of the High Commissioner for Human Rights (n.d) UN General Assembly. 1990. Convention on the Rights of the Child. General Assembly resolution 44/25. http://www.ohchr.org/en/ professionalinterest/pages/crc.aspx. Accessed August 12, 2013). 10. Safe Youth Worldwide (2005). Scaling Up HIV Prevention Programs for Youth: The Essential Elements (http://www.plannedparenthood.org/nyc/files/NYC/ScalingUpSYW_online.pdf) 11. Multinacional: Hargreaves et al., 2008a, Gray I; Hargreaves and Glynn, 2002; World Bank, 2002 cited in Global Campaign for Education, 2004, Gray V; South Africa: Hargreaves et al., 2008b, Gray II; Bärnighausen et al., 2007, Gray IV; Pettifor et al., 2008a, Gray IV; Ethiopia: Bradley et al., 2007, Gray IIIb; Zambia: Michelo et al., 2006, Gray IIIb; Malawi: Clark et al., 2009, Gray IIIb; India, Cambodia: Greener and Sarkar, 2010, Gray IV; Lao PDR: Schyareun et al., 2011, Gray IV; Burkina Faso, Ghana, Malawi, Uganda: Biddlecom et al., 2007, Gray IV; Zimbabwe: Gregson et al, 2004 12. Ethiopia, Ghana, Kenya, Malawi, Mozambique: The World Bank and UNICEF, 2009, Gray IIla; Tanzania, Kenya, Uganda: Burns et al., 2003; UNICEF, 2005; Deininger, 2003; Bundy and Kattan, 2005, cited in Global Coalition on Women and AIDS, year not specified, Gray V

13. Zimbabwe: Hallfors et al, 2011, Gray II; Kenya: Cho et al., 2011, Gray II; Zambia: Chatterji et al., 2010: 139, Gray IIIb 14. Malawi: Baird et al., 2012, Gray II

15. Véase UNESCO, 2009b; Pulerwitz et al., 2006; Barker et al., 2010b; Peacock, 2009

16. Multinacional: Johnson et al., 2011, Gray I; UNESCO, 2009b, Gray I; Kirby et al, 2007a; Kirby et al., 2007b; Kirby et al., 2006; Kirby, 2009, Gray I; Sub-Saharan Africa: Michielsen et al., 2010, Gray I; Mavedzenge et al., 2010, Gray I; Swaziland: Burnett et al., 2011, Gray II; Tanzania: Ross et al., 2007a: 1952, Gray II; Bahamas: Chen et al., 2010b, Gray II; South Africa: Reddy and James, 2003, Gray IIla; Uganda: Aggleton et al., 2000, Gray IIla; Kenya: Maticka-Tyndale, 2010, Gray IIla; Agbemenu and Schlenk, 2011, Gray IIIb; Thailand: Ishikawa et al., 2011a, Gray IIla; Brazil: Andrade et al., 2009, Gray IIIb; Gauri et al., 2007, Gray IV; Mexico: Pick et al., 2007, Gray IV

17. Mavedzenge, Doyle and Ross, 2010

18. Comprehensive Sexuality Education: The Challenges and Opportunities of Scaling Up, UNESCO, 2012

19. Multinacional: UNESCO, 2009b, Gray I; Uganda: Shuey et al., 1999 cited in James-Traore et al., 2004, Gray IIla; Yemen: Al-Iryani et al., 2011, Gray IIla; Cameroon: Arcand \& Wouabe 2010, Gray IV

20. 80 country review: Ross et al., 2006, Gray IIIb; Mozambique: Melo et al., 2008, Gray IIIb; Madagascar: Neukom and Ashford, 2003, Gray IIIb

21. Revisión multinacional: Kennedy et al., 2010b, Gray I; Tan- zania, Kenya, Trinidad: Voluntary HIV-1 Counseling and Testing Efficacy Study Group, 2000, Gray II; Rwanda, Kenya, Tanzania, Trinidad, Uganda: Denison et al., 2008, Gray II; Bunnell et al., 2008, Gray IIIb; Zimbabwe: Cremin et al., 2010, Gray IIla; Gregson et al., 2002, Gray IIIb; Mozambique: Mola et al., 2006, Gray IIla; South Africa: Leon et al., 2010a, Gray Illa; Pettifor et al., 2010, Gray IIIb; Kenya: Huchko et al., 2011, Gray IIIb; Dominican Republic: Sears et al., 2008, Gray IIIb; Rwanda: Allen et al., 2003, Gray IIIb; Tanzania: Maman et al., 2001b: 597, Gray IIIb; Botswana: Creek et al., 2006, Gray IV

22. Ethiopia: Bradley et al., 2008a, Gray IIIb; Haiti: Peck et al., 2003, Gray IIIb; Kenya: Liambila et al., 2009, Gray IIIb; South Africa: Kharsany et al., 2010a, Gray IV

23. Botswana, Kenya, Malawi, South Africa, Zimbabwe, Brazil, India, Thailand, United States: Cohen et al., 2011, Gray II; Botswana, Kenya, Rwanda, South Africa, Tanzania, Uganda, Zambia: Donnell et al., 2010, Gray IIla; Sub-Saharan Africa: Attia et al., 2009: 1401, Gray V; Switzerland: Cohen et al, 2009, Gray V 24. South Africa: Kim et al., 2007a; Kim et al., 2009a, Gray IIIb; Kenya: Kilonzo et al., 2009a, Gray IIIb; Siika et al., 2009, Gray IIIb; Malawi, Zambia: Keesbury and Askew, 2010, Gray V 25. Cochrane review: Vidanapathirana et al., 2005, Gray I; Tanzania: Mossdorf et al., 2010, Gray IIIb; Kenya: Marum et al., 2008, Gray IIIb

26. Lovelife. 2012. Talking Points 2012: A study on HIV, sexual risk behavior, and access to opportunity among young people in South Africa evaluating LoveLife's impact on the life choices and risk tolerance of young people. South Africa. www.lovelife.org.za. 27. Multinacional: Bertrand et al., 2006, Gray I; Rwanda: Neukom and Ashford, 2003, Gray IIla; Cameroon: Neukom and Ashford, 2003, Gray IIla; Zimbabwe: Kim et al., 2001, Gray IIIa; Zambia: Underwood et al., 2001; Gray IIIb; Nepal, Brazil, Senegal: Geary et al., 2007, Gray IV; Uganda: Adamchack et al., 2007, Gray V

28. Thailand: Apinundecha et al., 2007, Gray Illa; UNAIDS, 2007b; Viravaidya et al., 2008, Gray IIIb; Vietnam: Nyblade et al., 2008, Gray IIIb; China: Yang and Zhang, 2004, Gray IIIb; Nigeria: Fakolade et al., 2010; Babaloba et al., 2009, Gray IIIb; Malawi: Berendes and Rimal, 2011:224, Gray IIIb; Ghana: Boneh and Jaganath, 2011, Gray IV

29. Nicaragua: Solarzano et al., 2008, Gray Illa; Brazil: Pulerwitz et al. 2006, Gray IIIb

30. Zambia: Morel-Seytoux et al., 2010, Gray IIIb; South Africa: Usdin et al., 2005, cited in Rottach et al., 2009, Gray IV; India: Pelto and Singh, 2010, Gray IIIb

31. Greene, M. and A. Levack. 2010. Synchronizing Gender Strategies: A Cooperative Model for Improving Reproductive Health and Transforming Gender Relations. Washington, DC: Population Reference Bureau, Interagency Gender Working Group. www. igwg.org, (p.vi).

32. South Africa: Dreyer, 2001 cited in James-Traore et al., 2004, Gray IIIb; Ghana, Malawi: USAID, 2008a, Gray IIIb

33. South Africa: Jewkes et al., 2006b; Gray II; Colvin, 2009, Gray IIIb; Jewkes et al., 2010c, Gray IV; Ethiopia: Pulerwitz et al., 2010a; Gray IIla;

34. South Africa: Jewkes et al., 2006b, Gray II; Colvin, 2009, Gray IIIb; India: Verma et al., 2008, Gray IIla; Tanzania: Maganja 
et al., 2007, Gray IIla; Botswana: Norr et al., 2004; Gray Illa; Brazil: Pulerwitz et al., 2006, Gray IIIb

35. Uganda: Kairania et al., 2010, Gray IIlb; Tanzania: Maman et al., 2001a, Gray IV; Maman et al., 2001b, Gray IV; Rwanda, Zambia: Allen et al., 2007b, Gray V; Kenya: Sarna et al., 2009, Gray V 36. Malawi: Bello et al., 2011a, Gray Illb; Uganda: Slaymaker et al., 2009, Gray IIlb; Zambia: Sandøy et al., 2007, Gray IV; Zimbabwe: Gregson et al., 2006, Gray IV

37. Uganda, Tanzania, Ghana, Botswana: African Youth Alliance, 2007, Gray Illa; Zimbabwe: Terry et al., 2006, Gray IIla

38. Tanzania: Kamenga et al., 2001, Gray II; Zimbabwe: Cremin et al., 2010, Gray IIla; India: Solomon et al., 2006, Gray IIIb 39. South Africa: Kalichman et al., 2008, Gray II; Wechsberg et al., 2006, Gray IIla; Kenya: Mackenzie et al., 2008, Gray IIla; Luseno and Wechsberg, 2009, Gray IIIb

40. South Africa: Phetla et al., 2008, Gray IIIb; Ghana: Wolf and Pulerwitz, 2003, Gray IIIb; Uganda: Damalie, 2001, Gray IV

41. Australia: Paxton, 2002, Gray IV; South Africa: Phetla et al., 2008, Gray V

42. IOM, NAS, 2001; Cochrane Collaborative Review Group on HIV Infection and AIDS, 2004, Gray I; Multinacional: Davis and Weller, 1999, Gray I; Eastern and Southern Africa: Hughes et al., 2012, Gray IIlb; Brazil: UNAIDS, 2008, Gray V

43. PATH and UNFPA, 2006; Drew et al., 1990 cited in Hoke et al., 2007, Gray II; Feldblum et al., 2001; Fontanet et al., 1998; French et al., 2003 cited in Dias et al., 2006, Gray II; Trussell et al., 1994 cited in Fernandez et al., 2006, Gray II; Nelson, 2007 in Lancet; Gray V

44. Meta-analysis: Scott-Sheldon et al., 2011b, Gray I;

45. Tanzania, Cameroon, Ghana, China, Indonesia, Thailand,

Carribbean, Mexico, Central America: Charania et al., 2011, Gray IIla; Kenya: Papo et al., 2011, Gray IIIb

46. Brazil: Barbosa et al., 2007: 265, Gray Illb; Kenya: Thomsen et al., 2006, Gray IIlb; Brazil, South Africa: Dowdy et al., 2006, Gray IIlb; Madagascar: Hoke et al., 2007, Gray IIlb; China: Liao et al., 2011a and b, Gray Illb; Zimbabwe: Napierala et al., 2008, Gray IIIb

47. Zambia: Mark et al., 2007, Gray II; Ethiopia: Aklilu et al., 2001, Gray IIla

48. South Africa: Wechsberg et al., 2010, Gray IIlb; Zimbabwe: Callegari et al., 2008, Gray IIIb

49. 18 African countries: Cleland et al., 2006b, Gray IV; South Africa: Maharaj and Cleland, 2006, Gray V; Africa: Cleland et al., 2006a, Gray V; Brazil: Juarez and Martin, 2006, Gray V

50. Mexico: Zellner et al., 2006, Gray IIIb; Cameroon, Rwanda: Neukom and Ashford, 2003, Gray IV

51. Brazil, North America, New Zealand: Knerr et al., 2009, Gray IV; Cambodia, Namibia, South Africa, Senegal, Zimbabwe, Sri Lanka, Mongolia, India and the UK: Philpott et al., 2006, Gray V 52. Malawi: Zulu and Chepngeno, 2003, Gray IIlb; South Africa: MacPhail et al., 2007, Gray IV; Uganda: Williamson et al., 2006, Gray IV; India, Thailand, Uganda: McGrath et al., 2007, Gray V 53. Uganda: Singh et al., 2003a, Gray Illb; Ethiopia, Tanzania, Zambia: Nyblade et al., 2003, Gray Illb; Zimbabwe: Feldman and Masophere, 2003: 165, Gray IIlb; 18 countries in Sub-Saharan Africa: Cleland et al., 2006a, Gray V

54. Weiss et al., 1996 cited in Weiss and Gupta, 1998; Kaufman et al., 2002

55. Nota: en algunos casos, los microcréditos pueden incrementar la violencia contra las mujeres si la intervención no se diseña con cuidado y no resulta adecuada para el contexto local. (Schuler et al., 1998; Gupta et al., 2008a; Dunbar et al. 2010).

56. South Africa: Pronyk et al., 2008a, Gray II; Kaufman et al., 2002, Gray IV; Haiti: Longuet et al., 2009, Gray IIIb; Republic of Congo: Boungou, 2007, Gray V

57. Zimbabwe: Corbett et al., 2006, Gray II; Democratic Republic of Congo, Rwanda, Burundi, Republic of Congo, Nigeria: Van der Borght et al., 2010, Gray IV

58. Uganda: Kumakech et al., 2009, Gray II; Rwanda: Brown et al., 2009, Gray Illa; Kenya, Tanzania: Nyangara et al., 2009a, Gray IV

59. China: Zhao et al., 2010b, Gray Illb; South Africa: Boon et al., 2009, Gray IV; Rwanda: Irwin et al., 2009: 49 citing Binagwaho et al., 2008, Gray V; Botswana: Kidman et al., 2007, Gray V; Zimbabwe: Miller et al., 2011a: 37, Gray V; Kenya: Thurman et al., 2012, Gray IIIb

60. Chatterji et al., 2005; Ayieko, 1998; HRW, 2003c; Birdthistle et al., 2008; Gregson et al., 2005

61. Uganda: Ssewamala et al., 2009, Gray II; Ssewamala et al., 2010, Gray IV; Kenya: Skovdal et al., 2010a: 7, Gray V; South Africa: Adato and Bassett, 2008 citing Samson et al., 2004, Gray IV; Freeman et al., 2006, Gray V; Malawi, Zambia: Adato and Bassett, 2008 citing Schubert et al., 2007, Gray IV

62. Kenya, Tanzania: Nyangara et al., 2009b, Gray IV; Tanzania: Wallis et al., 2010, Gray IV

63. UNICEF, 2013, Experiences from the Field: HIV Prevention among Most at Risk Adolescents in Central and Eastern Europe and the Commonwealth of Independent States, page v.

64. India: Halli et al., 2006, Gray II; Swendeman et al., 2009, Gray Illa based on Basu et al., 2004 and Saha, 2008; Ramesh et al., 2010, Ng et al., 2011, Pickles et al., 2010, Gray Illb; China: Lau et al., 2007a, Gray IIla; Wu et al., 2007b, Gray IIlb 65. Meta-analysis in Sub-Saharan Africa, East and Central Asia, Latin America: Medley et al., 2009a, Gray I

66. La aplicación de las políticas varía en la práctica. Documentos recientes con políticas relativas al uso del preservativo en todos los casos, por ejemplo en Camboya (Lowe, 2002), sugieren que determinados aspectos de las políticas relativas al uso del preservativo en todos los casos pueden restar autoridad a los trabajadores sexuales y violar los derechos humanos. Algunos han sugerido que los estudios de Kerrigan de 2004 y 2006 , junto con el estudio de Pisey de 2008 y el de Morisky y Tiglao de 2010 no deberían aplicarse por esta razón. La campaña relativa al uso del preservativo en todos los casos llevada a cabo en Tailandia "podría haber afectado negativamente a los trabajadores sexuales marginados con el incremento de la corrupción, las redadas policiales y las pruebas obligatorias de detección del VIH" (Shannon et al., 2009: 659). "Es esencial que todos los programas adopten un enfoque orientado a los trabajadores sexuales y permitan a estos determinar colectivamente qué papel deberían desempeñar los propietarios de los burdeles en los programas de intervención relativos al VIH/SIDA" (ONUSIDA, 2011f: 12).

67. Dominican Republic: Kerrigan et al., 2006; Kerrigan et al., 
2004, Gray IIla; Philippines: Morisky and Tiglao, 2010; Morisky et al., 2010; Chiao et al., 2009; Ang and Morksy, 2011, Gray IIlb; China: Hong et al., 2008, Gray IV

68. Mexico: Patterson et al., 2008, Gray Illa; China: Rou et al., 2007, Gray IIlb; Guatemala: Sabido et al., 2009, Gray IIIb; Mozambique: Lafort et al., 2010: 146, Gray IIlb; Brazil: Lippman et al., 2010, Gray V

69. China: Li et al., 2006, Gray Illa; Kenya: Ngugi et al., 2007, Gray V; South Africa: Stadler and Delany, 2006, Gray V

70. Armenia: Markosyan et al., 2010, Gray II; Kenya: Bandewar et al., 2010, Gray IIIb; India: Gooptu and Banyopadhyay, 2007, Gray V; Pillai et al., 2008, Gray V; Argento et al., 2011; Gray V; Systematic review: Shahmanesh et al., 2008; Padian et al., 2011b, Gray V

71. Senegal: Leonard et al., 2000, Gray IIIb; India: Lipovsek et al., 2010, Gray IIIb

72. Metzger and Navaline, 2003 cited in Strathdeee et al., 2006; Demaan et al., 2002 cited in Strathdee et al., 2006; Metzger et al., 2003 cited in Strathdeee et al., 2006; Ball et al., 1988 cited in Strathdee et al., 2006; Bruce, 2010; Roberts et al., 2010, Additional evidence: Multinacional: Kimber et al., 2010, Gray I; Moses et al., 1994 cited in IOM 2007; Serpellini and Carrieeri, 1994 cited in IOM, 2007, Gray IIla; Larney and Dolan, 2009, Gray IIIb; Cochrane review: Mattick et al., 2008, Gray I; Mattick et al., 2009, Gray I; Gowing et al., 2011; Gray IIlb; Jurgens et al., 2009b, Gray IIIb; Malaysia: Schottenfeld et al., 2008, Gray I; United States: McCarthy et al., 2005, Gray IIlb; United States, Europe, Australia: Beusekom and Iguchi, 2006, Gray IV; Ukraine, Pakistan, Kenya: Strathdee et al., 2010, Gray IIlb; Taiwan: CDC, Taiwan cited in Tsai et al., 2010, Gray IIIb

73. United States, Canada, Europe, Nepal, Russia: Wodak and Cooney, 2006: 802. Gray I; China: Wu et al., 2007a, Gray II; Chen et al., 2007c, Gray IIIb; Chawarski et al., 2011, Gray Illa; India: Sharma et al., 2009, Gray IIIb; Australia: Topp et al., 2011, Gray IIIb; Canada: Kerr et al., 2010b, Gray IIlb; Bangladesh: Guinness et al., 2009, Gray IIlb; Brazil: PHR, 2007b, Gray IIIb; Meta-analysis: Palmateer et al., 2010, Gray Illb; Smyrnov, P., Broadhead, R.S., Datsenko, O., Matiyash, O. (2012). Rejuvenating harm reduction projects for injection drug users: Ukraine's nationwide introduction of peer-driven interventions. International Journal of Drug Policy, 23, 141-147.

74. Meta-analysis in Sub-Saharan Africa, East and Central Asia, Latin America: Medley et al., 2009a, Gray I; Vietnam: Hammett et al., 2012, Gray IIIb

75. Cochrane review: Meader et al., 2010, Gray Illb; Kazakhstan: Gilbert et al., 2010, Gray Illa; Russia: OSF, 2012, Gray IIIb

76. Ukraine: Booth et al., 2009, Gray IIIb; South Africa: Needle et al., 2008; Parry et al., 2008; Parry et al., 2009, Gray Illb; Estonia: Wilson et al., 2007, Gray V

77. Carencia observada globalmente en las niñas de 15 a 19 años, pues el porcentaje de estas niñas en las escuelas es muy reducido (Haberland y Rogow, 2007). Carencia observada también, por ejemplo, en la República Democrática Popular Lao (Sychareun et al. 2011); Pakistán (Farid-ul-Hasnain y Krantz, 2011); Camerún (Tsala Dimbuene y Kuate Defo, 2011); Jamaica (Ishida et al., 2011); Yemen (Al-Serouri et al., 2010); Zambia (Carnevale et al., 2011); Nepal (Upreti et al., 2009); Nicaragua (Manji et al.,
2007); Etiopía (Alemu et al., 2007; Erulkar et al., 2006); más de 30 países de África y cuatro países de Asia (Dixon-Mueller, 2009).

78. Carencia observada, por ejemplo, en Antigua y Barbados; Bahamas; Bolivia; Colombia: Costa Rica; Chile; Dominica; Ecuador; El Salvador; Guyana; Haití; Honduras; Jamaica; México; Nicaragua; Panamá; Paraguay; Perú; Venezuela; la República Dominicana; Santa Lucía; Suriname; Trinidad y Tabago; y Uruguay (DeMaria et al., 2009); la India (McManus y Dhar, 2008). 79. Carencia observada, por ejemplo, en Kenya (Agbemenu y Schlenk, 2011); Zimbabwe (Ferrand et al., 2011); Tanzania (Ferrand et al., 2010); la India, Botsuana, Kenya, Malawi, Mozambique, Ruanda, Tanzania, Tailandia, Trinidad, Uganda, Zambia y Zimbabwe (McCauley, 2004) y Sudáfrica (Human Rights Watch, 2003a).

80. Carencia observada globalmente (Malhotra et al., 2011; CHANGE, 2009, Ezer et al., 2006).

81. Carencia observada, por ejemplo, en 11 países pertenecientes al Programa de Encuestas Demográficas y de Salud (Hargreaves y Glenn, 2002; Banco Mundial, 2002 citado en Global Campaign for Education, 2004).

82. Carencia observada, por ejemplo, en un examen de 45 estudios cuantitativos y cualitativos en África Subsahariana (Hope, 2007); Liberia (Atwood et al., 2011); Botsuana, Namibia y Suazilandia (Cockcroft et al., 2010); Botsuana, Malawi y Mozambique (Underwood et al., 2001); Tanzania (UNICEF, Tanzania et al., 2011a; Silberschmidt y Rasch, 2001); Zimbabwe (Munjoma et al., 2010); Perú (Sandoval et al., 2009); Camerún (Hattori y DeRose, 2008); Uganda (Nobelius et al., 2011; Samara, 2010); Sudáfrica (Ott al., 2011; Jewkes et al., 2002 citado en Jejeebhoy y Bott, 2003); Sudáfrica y Uganda (Geary et al., 2008; Katz y LowBeer, 2008); Burkina Faso, Ghana, Malawi y Uganda (Bankole et al., 2007); Botsuana (Médicos en pro de los Derechos Humanos, 2007a); Kenya (Longfield et al., 2004); Ghana (Goparaju et al., 2003); Zimbabwe (Gregson et al., 2002).

83. Carencia observada, por ejemplo, en Sudáfrica (Jewkes et al., 2010b); Egipto (Nada y Suliman, 2010); la India (Bal et al., 2010); Namibia, Suazilandia, Uganda, Zambia y Zimbabwe (Brown et al., 2009).

84. Carencia observada, por ejemplo, en Etiopía, Kenya, Malawi, Zambia y Sudáfrica (Keesbury y Askew, 2010); Filipinas (Ramiro et al., 2010); Sudáfrica (Human Rights Watch, 2003a).

85. Carencia observada, por ejemplo, en Irán (Claeson, 2011); Ucrania (Izenberg y Altice, 2010); Viet Nam (Nguyen et al., 2012, resumen); Indonesia (Afriandi et al., 2010); Tailandia (Kerr et al., 2010c); China, Rusia, Viet Nam, Ucrania y Malasia (Wolfe et al, 2010); México (Moreno et al., 2010); Tailandia, Indonesia, Bangladesh, Myanmar, la India y Nepal (Sharma et al., 2009); y en general (Piot et al., 2008, Mattick et al., 2003; Gowing et al., 2005 citado en OIM, 2007).

86. Carencia observada, por ejemplo, en Azerbaiyán, Georgia, Kirguistán, Rusia y Ucrania (OSI, 2009); Azerbaiyán, Kazajstán, Kirguistán, Tayikistán, Turkmenistán y Uzbekistán (UNODC, 2010a); China (Jia et al., 2010; Human Rights Watch, 2010b; Sullivan y Wu, 2007: 121, Liu et al., 2006a: 119); Camboya (Human Rights Watch, 2010a); Ucrania (Strathdee et al., 2010); Sudáfrica (Parry et al., 2010); Tailandia (Hayashi et al., 2009); Viet Nam 
(Thanh et al., 2009a); y en general (Wolfe et al., 2010; Jurgens et al., 2010; Gowan et al., 2008); Camboya, China, Malasia y Viet Nam (OMS et al., 2011b).

87. Carencia observada, por ejemplo, en Ucrania (Busza et al., 2010; Teltschik et al., 2008)

88. Carencia observada, por ejemplo, en Hungría (Gyarmathy et al., 2011b); Rusia (Niccolai et al., 2010); Sudáfrica (Scheibe et al., 2011, Parry et al., 2010) y Asia Central (Thorne et al., 2010). 89. Carencia observada, por ejemplo, en Viet Nam (Hammett et al., 2010); China (Jiang et al., 2010); la India (Solomon et al., 2010a); Brasil (Nappo et al., 2011); globalmente (Roberts et al., 2010); Rusia (Toussova et al., 2009); Viet Nam (Nguyen y Scannapieco, 2008, Go et al., 2006); Brasil (Oliveira, 2007); Ucrania (Strathdee et al., 2010); Sudáfrica (Parry et al., 2010); y en general (OIM, 2007, Roberts et al., 2010).

90. Carencia observada, por ejemplo, para Rusia (Abdala et al., 2011, Sarang et al., 2010); Camboya (Human Rights Watch, 2010); Kirguistán, Kazajstán y Tayikistán (Shapoval y Pinkham, 2011); Sudáfrica (Weschberg et al., 2008 citado en El-Bassel et al., 2010; Parry et al., 2009) y Kenya y Tanzania (Nieburg y Carty, 2011).

91. Carencia observada, por ejemplo, en Tailandia (Vuttanont et al., 2006); el Brasil (Mane et al., 2001, Juarez y Martin, 2006);

Sudáfrica (Moyo et al., 2008); Mozambique (Machel, 2001). 92. Carencia observada, por ejemplo, globalmente (Bruce et al., 2011).

93. Carencia observada, por ejemplo, en Kenya (Njue et al., 2009).

94. Carencia observada en África Subsahariana (Fatusi e Hindin, 2010); Etiopía (Lindstrom et al., 2010); numerosos países (UNESCO, 2009b).

95. Carencia observada, por ejemplo, en Mozambique (Hayford y Agadjanian, 2010); Sudáfrica (Scorgie et al., 2011; Mqhayi et al., 2003 citado en Mantell et al., 2005); Kenya (Brady et al., 2009); Brasil (Dias et al., 2006); Uganda (Wanyenze et al., 2011a; Green et al., 2001); en general (Hoffman et al., 2004; Green et al., 2001; Okunlola et al., 2006; Mathews y Harrison, 2006).

96. Carencia observada, por ejemplo, en Kenya (Mung'ala et al., 2006); Sudáfrica, los Estados Unidos de América, y Nigeria (Mantell et al., 2001).

97. Carencia observada, por ejemplo, en Senegal, Burkina Faso, Nigeria, Kenya, Namibia y Suazilandia (Winskell et al., 2011a). 98. Carencia observada, por ejemplo, en Uganda (Wawer et al., 2009); África Subsahariana (Hallett et al., 2008a); Kenya (Agot et al., 2007); Sudáfrica (Taljaard et al., 2008); Uganda y Zimbabwe (Matovu et al., 2007); Kenya, Ruanda, Sudáfrica y Zambia (Baeten et al., 2010).

99. Carencia observada, por ejemplo, en Kenya, Namibia, Sudáfrica, Suazilandia y Uganda (AVAC et al, 2010); y Sudáfrica y Zimbabwe (Mavedzenge et al., 2011b).

100. Carencia observada, por ejemplo, en Zimbabwe (Ferrand et al., 2011).

101. Carencia observada, por ejemplo, en Zambia (Bond, 2010); Camerún (Njozing et al., 2010); Viet Nam (Nam et al., 2010); Malawi (Namakhoma et al., 2010).

102. Carencia observada, por ejemplo, globalmente (Jurgens, 2007a); Sudáfrica (Venkatesh et al., 2011a); Pakistán (Hussain et al., 2011); Tanzania (Mmbaga et al., 2009); y Zimbabwe (Sherr et al., 2007).

103. Carencia observada, por ejemplo, en 22 países de la región del Mediterráneo Oriental (Hermez et al., 2010); Zimbabwe (Sambisa et al., 2010); la India (Joseph et al., 2010); Kenya (Karau et al., 2010); Sudáfrica (Groves et al., 2009); Botsuana (Médicos en pro de los Derechos Humanos, 2007a y Weiser et al., 2006a); China (Li et al., 2007); y Ucrania (Yaremenko et al., 2004).

104. Carencia observada, por ejemplo, en Sudáfrica (Kelvin et al., 2008; Ngema et al., 2008; Harrison, 2008); Chile (Cianelli et al., 2008); China (Zhou, 2008); América Latina (Parodi y Lyra, 2008); y Zimbabwe (Feldman y Masophere, 2003).

105. Carencia observada, por ejemplo, en 29 países de África y América Latina (Clark et al., 2006); un examen de más de 150 estudios (Collins y Rau, 2000; Gupta et al., 2003 citado en Gillespie y Kadiyala, 2005); Sudáfrica (Bhana y Pattman, 2011); Zimbabwe (Feldman y Masophere, 2003); Etiopía, Malawi, y Haití (Mathur et al., 2003); y Tanzania (Silberschmidt y Rasch, 2001). 106. Carencia observada, por ejemplo, en Nigeria (Etiebel et al., 2012); Malawi, Namibia y Botsuana (Beyrer et al., 2010b); China (Zhou, 2006): la India (Guterrez et al., 2010; Hernandez et al., 2006); y Nicaragua (Beyrer et al., 2010 citado en OMS et al., 2011b).

107. Carencia observada, por ejemplo, en Uganda (Miller et al., 2011b); Haití (Fawzi et al., 2010); Viet Nam (Phinney, 2008);

Brasil (Hebling y Guimaraes, 2004); Serbia (Bernays et al., 2010) y Sudáfrica (Susser y Stein, 2000).

108. Crencia observada, por ejemplo, en Zambia (Chimuka, 2002).

109. Carencia observada, por ejemplo, en Uganda (Wawer et al., 2012; Kajubi et al., 2011); la India (Solomon et al., 2010a); China (Li et al., 2011a; Yun et al., 2011); Sudáfrica y Zimbabwe (Mavedzenge et al., 2011); Sudáfrica (Mah, 2010); Nigeria (Adebayo et al., 2011; Oydiran et al., 2010); Botsuana (Thomas y Lungu, 2010; Foster et al., 2010a); Sudáfrica (Tanser et al., 2011); Mozambique (Noden et al., 2009); Tanzania (Exavery et al., 2011); Etiopía (Molla et al., 2008); la Indica (Chatterjee y Hosain, 2006). Zimbabwe (Callegari et al., 2008; Feldman y Masophere, 2003); México (Hirsch et al., 2007; Pulerwitz et al., 2001); Kenya y Zambia (Glynn et al., 2001; Glynn et al., 2003); Kenya (Kaiser et al., 2011); Zambia (Clark, 2004) y globalmente (Green et al., 2009) y de lugares del estudio HPTN 052 (Eshelman et al., 2011).

110. Carencia observada, por ejemplo, en Zimbabwe (Ferrand et al., 2011); Uganda (Kayiki y Forste 2011); Taiwán (Tung et al., 2010); Malasia (Anwar et al., 2010); Sudáfrica (Tenkorang et al., 2011; Anderson et al., 2007, Stadler et al., 2007); y Burkina Faso, Ghana, Malawi y Uganda (Biddlecom et al., 2007).

111. Carencia observada, por ejemplo, en Camerún (Arcand \& Wouabe 2010); Tanzania (UNICEF, Tanzania et al, 2011a); Tailandia (Ishikawa et al., 2011a); Bolivia, Chile y México (López Torres et al., 2010).

112. Carencia observada, por ejemplo, en África Subsahariana (Sommer, 2011); Tanzania (Sommer, 2010) y Pakistán (Ali y Rizvi, 2010).

113. Carencia observada, por ejemplo, en Sudáfrica (Cluver et al., 2011). 
114. Carencia observada, por ejemplo, en Camboya, la India, Kenya, Tanzania y Etiopía (Messer et al., 2010); Haití (Surkan et al., 2010); Zimbabwe (Kembo, 2010; Nyamukapa et al., 2010); Ruanda (Betancourt et al., 2011); Ruanda (Thurman et al., 2008a); Sudáfrica (Van der Heijden y Swartz, 2010; Cluver et al., 2007, Cluver y Gardner, 2007); China (Xu et al., 2010a y b; Zhao et al., 2010a; Zhang et al., 2009a; He y Ji, 2007).

115. Carencia observada, por ejemplo, en una revisión sistemática Sherr, 2008); Sudáfrica (Hill et al., 2008; y Zimbabwe (Nyamukapa y Gregson, 2005).

116. Carencia observada globalmente (Overs y Hawkins, 2011); Turkmenistán (Chariyeva et al., 2011); Suazilandia (Chipamaunga et al., 2010); Sudán (Abdelrahim et al., 2010); Somalia (Kriitmaa et al., 2010); y Pakistán (Khan et al., 2010) Afganistán (Todd et al., 2011a; Kiritbati (McMillan y Worth, 2010); y China (Zhang et al., 2011b).

117. Rotheram-Borus, M. J., D. Swendeman and G. Chovnick (2009). "The past, present, and future of HIV prevention: integrating behavioral, biomedical, and structural intervention strategies for the next generation of HIV prevention." Annu Rev Clin Psychol 5: 14367. http://www.ncbi.nIm.nih.gov/pubmed/19327028.

118. Yamey, G. (2011). “Scaling up global health interventions: a proposed framework for success.” PLoS Med 8(6): e1001049. http://www.ncbi.nlm.nih.gov/pubmed/21738450; Rottach, E., K. Hardee, R. Jolivet and R. Kiesel. 2012. Integrating gender into the scale-up of family planning and maternal, neonatal and child health programs. Washington, DC: Health Policy Project.; Hardee, K., L. Ashford, E. Rottach, R. Jolivet and R. Kiesel. 2011. The policy dimensions of scaling up health initiatives. Washington, DC: Health Policy Project. Draft; Global HIV Prevention Working Group (2007). Bringing HIV Prevention to Scale: An Urgent Global Priority. http:// www.globalhivprevention.org/pdfs/PWG-HIV_prevention_report_FINAL.pdf; Safe Youth Worldwide (2005). Scaling Up HIV Prevention Programs for Youth: The Essential Elements (http://www.plannedparenthood.org/nyc/files/NYC/ScalingUpSYW_online.pdf); Simmons, R. P. Fajans and P. Ghiron, editors. 2006. Scaling up health service delivery: from pilot innovations to policies and programmes. Geneva, Switzerland: World Health Organization; Hanson K, Ranson MK, Oliviera-Cruz V,Mills A (2003) Expanding access to priority health interventions: a framework for understanding the constraints to scaling-up. J Int Dev 15: 1-14.

119. Hanson K, Ranson MK, Oliviera-Cruz V,Mills A (2003) Expanding access to priority health interventions: a framework for understanding the constraints to scaling-up. J Int Dev 15: 1-14.

120.Mavedzenge SN, Luecke E, Ross DA. 2013. Effectiveness of HIV Prevention, Treatment and Care Interventions Among Adolescents: A Systematic Review of Systematic Reviews. UNICEF Technical Brief. New York. UNICEF. 


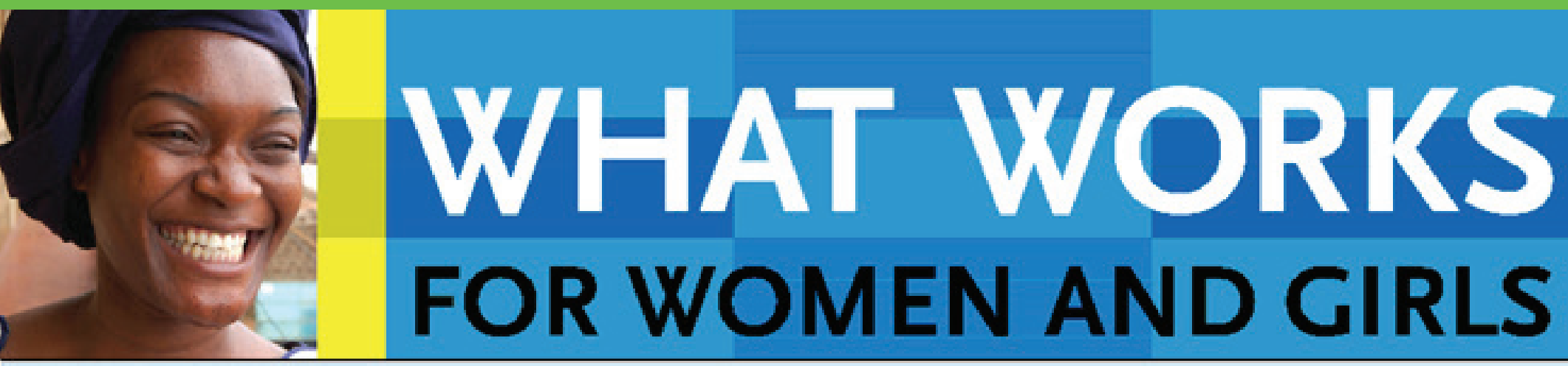

Pruebas de las intervenciones en materia de VIH/SIDA
$>$ El VHI y el SIDA afectan especialmente a mujeres y niñas.

> Para abordar el VIH/SIDA entre las mujeres y las niñas se requieren pruebas de intervenciones satisfactorias.

Esas pruebas se agrupan en un sitio $>$ www.whalworksforwomen.org

What Works for Women \& Girls cuenta con el apoyo del President's Emergency Plan for AIDS Relief (PEPFAR) y Open Society Foundations, y se está realizando bajo el auspicio del Health Policy Project and Evidence Project respaldado por la USAID, el Public Health Institute, y What Works Association, Inc. Puede obtenerse más información en www.whatworksforwomen.org o a través del correo electrónico mcrocegalis@gmail.com

La información proporcionada en este documento no es información oficial del Gobierno de Estados Unidos y no representa necesariamente las opiniones o posiciones de la Agencia de Estados Unidos para el Desarrollo Internacional. 